PLANEJAMENTO EXPERIMENTAL BOX-BEHNKEN PARA A REMOÇÃO ELETROCINÉTICA DE ALUMÍNIO DO LODO GERADO POR ESTAÇÃO DE TRATAMENTO DE ÁGUA

\title{
BOX-BEHNKEN EXPERIMENTAL DESIGN FOR ELECTROKINETIC REMOVAL OF ALUMINUM FROM DRINKING WATER TREATMENT SLUDGE
}

\author{
MARDER, Fernanda ${ }^{1}$; STÜLP, Simone ${ }^{1}$; HILGEMANN, Maurício ${ }^{1 *}$ \\ ${ }^{1}$ Centro Universitário UNIVATES, Programa de Pós-Graduação em Ambiente e Desenvolvimento, \\ Av. Avelino Talini, 171, CEP 95914-014, Lajeado - RS, Brasil \\ (fone: +55 5137147000, ramal 5957) \\ * Autor correspondente \\ e-mail: mauricio.hilgemann@univates.br
}

Received 14 February 2017; received in revised form 18 June 2017; accepted 18 June 2017

\begin{abstract}
RESUMO
A remediação eletrocinética é uma tecnologia emergente que pode ser usada para remover contaminantes de solos ou lodo pela aplicação de um campo elétrico. Neste trabalho,foram investigados os efeitos do potencial aplicado, tempo de eletrólise e quantidade de eletrólito sobre a remoção eletrocinética de alumínio presente em lodo gerado por Estação de Tratamento de Águas (ETA), usando o planejamento experimental estatístico Box-Behnken. A análise multivariada forneceu um modelo significativo, no qual a parte quadrática, em particular, foi a mais significativa, justificando, assim, as experiências de otimização. Como não houve aparente falta de ajuste (lack of fit), o modelo pode ser considerado válido e seus resultados interpretados mais detalhadamente.Os valores dos coeficientes- $\beta$ e sua significância indicam que os coeficientes mais significativos são os efeitos quadráticos do potencial aplicado e do tempo de eletrólise. A superfície de resposta mostrou um rendimento ótimo previsto de $60,5 \%$ para o potencial aplicado de $25 \mathrm{~V}$, tempo de eletrólise de $72 \mathrm{~h}$ e quantidade de eletrólito de $90 \%$.
\end{abstract}

Palavras-chave:quimiometria, Box-Behnken, remediação eletrocinética, ETA.

\section{ABSTRACT}

Electrokinetic remediation is an emerging technology that can be used to remove contaminants from soils or sludge by application of an electric field. Here, we investigated the effects of potential apllied, electrolysis time and electrolyte content upon electrokinetic removal of aluminum from drinking water treatment sludge using the statistical Box-Behnken Design. The analysis gave a significant model, in which the quadratic part in particular was significant, thus justifying the optimization experiments. Since there was no apparent lack of fit, the model could be considered valid and its results interpreted more thoroughly. The values of the $\beta$ coefficients, and their significance indicates that the most significant coefficients are the quadratic effects of potential applied and electrolysis time. The response surface showed an optimum predicted yield of $60.5 \%$ for a potential applied of $25 \mathrm{~V}$, an electrolysis time of $72 \mathrm{~h}$ and $90 \%$ electrolyte content.

Keywords: chemometrics, Box-Behnken, electrokinetic remediation, WTP 


\section{INTRODUÇÃO}

Os centros urbanos e o rápido crescimento populacional exercem grande demanda por água potável, o que exige a exploração de recursos hídricos e o desenvolvimento eficiente de um sistema de tratamento e distribuição de água ao grande público. As Estações de Tratamento de Água (ETA) convencionais são os meios de tratamento de água bruta, os quais envolvem os processos de coagulação, floculação, sedimentação, filtração e desinfecção (Ahmad et al., 2016).

Como subproduto do processo de tratamento de água, há a geração de lodo, composto por sólidos e precipitados químicos (Tartari et al., 2011), decorrente da adição de coagulantes, como os sais de ferro $\left(\mathrm{Fe}_{2}\left(\mathrm{SO}_{4}\right)_{3}\right.$ e $\left.\mathrm{FeCl}_{3}\right)$ e/ou alumínio $\left(\mathrm{Al}_{2}\left(\mathrm{SO}_{4}\right)_{3}\right.$, cloro-sulfato de alumínio parcialmente hidrolisado e policloreto básico de alumínio (PAC)) (Vaz et al., 2010). A concentração de sólidos nos decantadores varia entre $0,1 \%$ a $4 \%$; já na água de lavagem de filtro, a porcentagem é de $0,004 \%$ a $0,1 \%$. Normalmente, $\quad 75-90 \%$ desses valores representam sólidos suspensos e 20-35\% de compostos voláteis (Julio et al., 2009).

Devido ao processo de tratamento da água bruta, o lodo químico proveniente de ETA é constituído por um material extremamente gelatinoso, composto por hidróxidos de alumínio e ferro, partículas inorgânicas como argila e silte, colóides com coloração e microrganismos, incluindo plâncton (Moreira et al., 2009). O lodo possui uma característica bastante similar aos solos quando comparado ao lodo gerado em tratamentos de efluentes, ou seja, o nitrogênio e o carbono orgânicos são mais estáveis, menos reativos e em menores concentrações que lodos provenientes de esgotos (Portella et al., 2003).

As disposições inadequadas dos resíduos de ETA estão associadas aos aspectos visuais desagradáveis no manancial receptor e ao uso da água a jusante como fonte de abastecimento de outras comunidades e para o sistema de irrigação (Fadanelli e Wiecheteck, 2010). Na flora, o alumínio pode causar a redução da taxa de crescimento radicular de plantas sensíveis, afetando o alongamento e a divisão celular. Essa restrição causa a diminuição da capacidade da planta de obter água e nutrientes do subsolo, em virtude do enraizamento superficial, tornando-a, assim, menos produtiva e mais susceptível à seca (Ferreira et al., 2006). Em seres humanos, o alumínio pode ocasionar danos neurológicos, como perda de memória e um possível aumento na incidência da doença de Alzheimer (Baird, 2002). Consequentemente, é crescente a preocupação com o destino final do lodo de ETA, uma vez que, se disposto inadequadamente, pode prejudicar o meio ambiente e a saúde humana. A busca por soluções para atender à legislação e por uma destinação adequada é uma das preocupações atuais, conforme a NBR10004 (ABNT, 2004), que classifica o lodo como resíduo sólido, e a Lei n 12.305 (BRASIL, 2010), que institui a Política Nacional de Resíduos Sólidos. No entanto, alternativas vêm sendo buscadas para realizar o reaproveitamento deste material, dando-lhe um destino mais nobre e não só acondicionando-o em aterros controlados.

A técnica de remediação eletrocinética vem ganhando atenção nos últimos anos. O processo de remediação consiste na aplicação de uma corrente contínua de baixa intensidade entre os pares de eletrodos enterrados na zona contaminada ou potencial. A água subterrânea e/ou um fluido fornecido externamente fornecem a condutividade necessária para a realização do tratamento. Isso faz com que os contaminantes sejam conduzidos para os eletrodos por migração iônica e/ou eletro-osmose. Nos eletrodos, além da provável deposição catódica, o principal processo que ocorre é a eletrólise da água (Bard et al., 2008).

Os estudos envolvendo a remediação eletrocinética têm mostrado que o processo ocorre em todas as espécies que formam íons em ambientes aquáticos, podendo ser aplicado a vários contaminantes, tais como ânions e cátions inorgânicos; ácidos carboxílicos orgânicos, fenóis; compostos alifáticos e aromáticos, como alguns corantes sulfonados; detergentes e alguns pesticidas, como o paraquat e diquat (Lageman et al., 2005).

A técnica de remediação eletrocinética tem sido amplamente utilizada para separar e extrair os contaminantes de solos contaminados com metais pesados (Yu e Neretnieks, 1997; Sah e Chen, 1998; Page e Page, 2002; Wang et al., 2007; Kim et al., 2015; Rosestolato et al., 2015).

No entanto, poucos estudos têm sido realizados para a remoção de metais presentes em lodo gerado por Estações de Tratamento de Efluentes (ETE) e/ou ETA através desta técnica (Wang et al., 2005; Yuan e Weng, 2006; Ferri et al., 2009; Ebbers et al., 2015), e poucos são os 
trabalhos que investigam a remoção de metais pesados de ETA, em especial, de alumínio. (Cherifi et al., 2009; Cherifi et al., 2011; Cherifi et al., 2016) Nesses trabalhos, Cherifi et al. investigaram a influência de fatores como temperatura, quantidade de água e $\mathrm{pH}$ do lodo e presença de diferentes complexantes sobre a remoção eletrocinética de Al de lodos formados por ETA.

A metodologia de superfície de resposta, como o Box-Behnken (BBD), é uma ferramenta poderosa para a otimização dos parâmentos de um tratamento. O método gera um modelo matemático que consegue prever a condição ótima de operação de processos de remoção de poluentes (Tak et al., 2015).

Assim, o objetivo deste trabalho foi utilizar o planejamento BBD para avaliar a eficiência do método de remediação eletrocinética na descontaminação do lodo de uma ETA que utiliza sulfato de alumínio no processo de coagulação/floculação durante o processo de beneficiamento da água.

\section{MATERIAL E MÉTODOS}

\subsection{Amostragem do Lodo na ETA}

As amostras de lodo oriundo dos processos de coagulação/floculação e decantação foram coletadas em unidades de tratamento de água no estado do Rio Grande do Sul. As amostras foram acondicionados a $4^{\circ} \mathrm{C}$ até a sua utilização.

\subsection{Descontaminação dos Materiais}

Previamente às análises, todos os materiais foram lavados e descontaminados. Todo o material sem resíduos, vidro ou plástico, foi lavado com água e detergente e então foi mergulhado na respectiva solução ácida de descontaminação, permanecendo pelo período de no mínimo $24 \mathrm{~h}$. As vidrarias foram descontaminadas em solução aquosa de $\mathrm{HNO}_{3}$ $1 \%(\mathrm{v} / \mathrm{v})$, e os materiais plásticos foram postos em solução alcóolica contendo $\mathrm{HNO}_{3} 10 \%(\mathrm{v} / \mathrm{v})$. Após, o material era retirado da solução e enxaguado com água ultrapura.

\subsection{Limpeza e Regeneração dasMembranas}

A limpeza e regeneração das membranas catiônica e aniônica foi realizada com a imersão em solução de $\mathrm{HCl} 5 \%$ por 15 min e então em solução de $\mathrm{NaCl} 5 \%$ por 30 min. Antes de serem utilizadas, as membranas eram colocadas em solução de $\mathrm{Na}_{2} \mathrm{SO}_{4} 3 \%$ por $24 \mathrm{~h}$.

\subsection{Quantificação de Alumínio}

Previamente ao processo de digestão, a amostra de lodo foi filtrada a vácuo e seca em mufla a $60^{\circ} \mathrm{C}$ por $24 \mathrm{~h}$. O processo de digestão das amostras foi realizado de acordo com o método EPA 3050B (EPA, 1996), o qual consiste em uma digestão úmida com mistura oxidante de $\mathrm{HNO}_{3}-\mathrm{H}_{2} \mathrm{O}_{2}$.

Após a etapa de digestão, a quantidade de alumínio presente nas amostras foi analisada pela técnica de Espectometria de Absorção Atômica com Atomização por Chama (FAAS), utilizando o equipamento Pin AAcle 900T (Perkin Elmer).

\subsection{Delineamento Experimental}

Para otimizar as condições do processo de remoção de alumínio, foi utilizado o planejamento experimental Box-Behnken. Foram realizados 12 experimentos com 3 replicatas do ponto central. Os três fatores independentes foram analisados em três níveis de variação (níveis inferior, superior e médio) e estão apresentados na Tabela 1. A montagem da matriz experimental, bem como o tratamento de dados, foi realizada com o auxílio do software The Unscrambler X (CAMO).

Tabela 1. Fatores de controle e níveis para o planejamento BBD

\begin{tabular}{c|c|c}
\hline Fatores & $\begin{array}{c}\text { Nível } \\
\text { inferior (-1) }\end{array}$ & $\begin{array}{c}\text { Nível } \\
\text { superior } \\
(+\mathbf{1})\end{array}$ \\
\hline Potencial & 5,0 & 25 \\
aplicado (V) & 15 & 72 \\
Tempo (h) & 70 & 90 \\
Eletrólito (\%) & \\
\hline
\end{tabular}

O tratamento eletroquímico foi realizado com o arranjo experimental mostrado na Figura 1. Ele consiste em três seções de $4 \times 4 \times 4,5 \mathrm{~cm}$ confeccionadas em acrílico, com 
aproximadamente $72 \mathrm{~mL}$ de capacidade para cada compartimento. No centro, coloca-se a amostra a ser remediada e nas extremidades deste reator, são posicionados dois compartimentos, ambos contendo solução de $\mathrm{Na}_{2} \mathrm{SO}_{4} \quad 3 \%$. No compartimento anódico é inserida uma placa de titânio e rutênio $\left(70 \mathrm{TiO}_{2} / 30 \mathrm{RuO}_{2}, \mathrm{DSA}^{\circledR}\right.$ De Nora), que servirá como ânodo, enquanto no compartimento catódico é inserida uma placa de Ti, que servirá como cátodo. Nesta câmara é extraída a solução de $\mathrm{Na}_{2} \mathrm{SO}_{4} 3 \%$ juntamente com o contaminante.

Os eletrodos de $\mathrm{Ti}$ e $\mathrm{Ti} / \mathrm{Ru}$ foram escolhidos por serem inertes no meio e às condições aplicadas ao processo. Cada eletrodo possui uma área de $80 \mathrm{~cm}^{2}$, com $15 \mathrm{~cm}^{2}$ de contato com a solução de $\mathrm{Na}_{2} \mathrm{SO}_{4}$. Os eletrodos foram previamente lavados com água ultrapura (ASTM, 2011), imersos em solução de $\mathrm{HCl} 5 \%$ $(\mathrm{v} / \mathrm{v})$ por um período de 10 minutos e novamente lavados com água. Foi aplicada uma diferença de potencial (ddp) entre os eletrodos durante um período (Nadal EQ030), determinada de acordo com a matriz de planejamento, assim como a quantidade de eletrólito que foi adicionada ao lodo seco a fim de fornecer a condutividade ao meio reacional. Membranas catiônicas e aniônicas foram introduzidas entre a amostra contaminada e os compartimentos catódico e anódico, respectivamente. A membrana aniônica (Selemion AMV) e catiônica (Selemion CMV) possuíam 12,57 $\mathrm{cm}^{2}$ de área de contato, e espessura $100 \mu \mathrm{m}$. Ao final do processo de remediação eletrocinética, a amostra do lodo sofreu digestão para a posterior quantificação de Al residual.

A avaliação da eficiência do método foi realizada atravésda diferença da quantidade de Al após o processo de remoção eletrocinética, em relação à quantidade de alumínio do lodo de ETA in natura.

\section{RESULTADOS E DISCUSSÃO:}

$\mathrm{Na}$ Tabela 2 é apresentada a matriz experimental utilizada no planejamento BBD, assim como as respostas obtidas - remoção de alumínio - em cada um dos 15 experimentos relizados.
Tabela 2. Planejamento BBD: efeito do potencial aplicado $\left(X_{1}\right)$, do tempo de eletrólise $\left(X_{2}\right)$ e da quantidade de eletrólito $\left(X_{3}\right)$ na resposta obtida $(Y$, remoção de $A l)$

\begin{tabular}{c|c|c|c|c} 
Exp. $^{*}$ & $\boldsymbol{X}_{\mathbf{1}}$ & $\boldsymbol{X}_{\mathbf{2}}$ & $\boldsymbol{X}_{\mathbf{3}}$ & $\boldsymbol{Y}(\mathbf{\%})$ \\
\hline $1(5)$ & 0 & -1 & -1 & 42,4 \\
$2(8)$ & 0 & +1 & -1 & 35,8 \\
$3(7)$ & 0 & -1 & +1 & 35,3 \\
$4(15)$ & 0 & +1 & +1 & 53,1 \\
$5(3)$ & -1 & 0 & -1 & 38,7 \\
$6(12)$ & +1 & 0 & -1 & 46,9 \\
$7(6)$ & -1 & 0 & +1 & 48,2 \\
$8(14)$ & +1 & 0 & +1 & 43,4 \\
$9(11)$ & -1 & -1 & 0 & 56,4 \\
$10(1)$ & +1 & -1 & 0 & 36,0 \\
$11(4)$ & -1 & +1 & 0 & 44,0 \\
$12(10)$ & +1 & +1 & 0 & 52,4 \\
$13(9)$ & 0 & 0 & 0 & 24,0 \\
$14(2)$ & 0 & 0 & 0 & 28,4 \\
$15(13)$ & 0 & 0 & 0 & 36,8 \\
\hline
\end{tabular}

* Os números entre parênteses representam a ordem em que os experimentos foram realizados

O modelo multivariado obtido expressa o erro, os fatores significativos e a influência das correlações entre as variáveis independentes na resposta obtida. A equação quadrática para os termos significativos é apresentada na Equação 1 (fatores com $p>0,1$ foram excluídos):

$$
\begin{aligned}
Y= & 29,73+7,20 \cdot X_{1} \cdot X_{2}+6,10 \cdot X_{2} \cdot X_{3} \\
& +10,06 \cdot X_{1}^{2}+7,41 \cdot X_{2}^{2}
\end{aligned}
$$

Onde $X_{1}, X_{2}$ e $X_{3}$ correspondem ao potencial aplicado (V), ao tempo de eletrólise (h) e à quantidade de eletrólito (\%), respectivamente. $Y$ corresponde à remoção de alumínio (\%).

A análise de variância ANOVA mostrou que o modelo foi significativo $(p=0,05093)$, enquanto a falta de ajuste no modelo (lack of fit) não foi significativa $(p=0,8258)$.A falta de ajuste no modelo prova se o erro na previsão de resposta é principalmente devido à variabilidade experimental ou a uma forma inadequada do modelo. Se o valor de $p$ por falta de ajuste for menor do que 0,05 , significa que o modelo não descreve a forma verdadeira da superfície de resposta. Assim, o modelo pode ser confiável para descrever a superfície de resposta adequadamente.

Os efeitos lineares dos três fatores investigados não tiveram efeitos significativos ( $p$ $>0,1)$, enquanto os fatores quadráticos do potencial aplicado $(p=0,01143)$ e do tempo $(p=$ 
0,03494) mostraram-se significativos, assim como as interações entre as variáveis potencial aplicado e tempo $(p=0,03362)$ e tempo e quantidade de eletrólito $(p=0,05718)$. O coeficiente de determinação $\left(R^{2}\right)$ foi 0,8948 , explicando $89,48 \%$ da variabilidade da resposta. Pela Tabela 2, pode-se observar que as amostras centrais mostram bastante variação. É por isso que poucos efeitos no modelo são muito significativos, existindo uma grande quantidade de variabilidade experimental.

As superfícies de resposta mostradas na Figura 2 são baseadas na Eq. 1 e mostram a influência das variáveis sobre a remoção de alumínio, com uma variável sendo mantida constante em seu nível ótimo, enquanto se alteram as outras duas dentro do domínio experimental. As superfícies de resposta mostram, nas Fig. 2A-C, as interações entre potencial aplicado e tempo, potencial aplicado e quantidade de eletrólito e tempo e quantidade de eletrólito, respectivamente.

A superfície de resposta para a remoção de alumínio não apresenta nenhum pico dentro do domínio experimental. A remoção de alumínio aumenta com o aumento do potencial aplicado, à medida que o tempo de eletrólise e a quantidade de eletrólito aumentam para seus valores máximos, sugerindo que o ponto ótimo para o valor do potencial aplicado encontra-se no limite superior do domínio experimental. De fato, este mesmo comportamento observa-se para as outras variáveis estudadas.

A remoção máxima de alumínio estimada a partir da Eq. 1 foi de $60,5 \%$ em um potencial aplicado de $25 \mathrm{~V}$, durante $72 \mathrm{~h}$ e contendo $90 \%$ de eletrólito. Este resultado mostrou-se similar ao trabalho desenvolvido por Cherifi et al. (2016), no qual obtiveram remoção de $60 \%$ de alumínio coletado no compartimento catódico de uma célula de três compartimentos.

Cabe ainda enfatizar que estes resultados foram obtidos para um determinado tipo de amostra, sendo que estudos comprovam que a natureza do sólido possui forte efeito sobre o desempenho eletrocinético (Akretche, 2002).

\section{CONCLUSÕES:}

A análise multivariada forneceu um modelo significativo, no qual a parte quadrática, em particular, foi a mais significativa, justificando assim as experiências de otimização. Como não houve aparente falta de ajuste (lack of fit), o modelo pode ser considerado válido e seus resultados interpretados mais detalhadamente.

Os valores dos coeficientes- $\beta$ e sua significância indicam que os coeficientes mais significativos são os efeitos quadráticos do potencial aplicado e do tempo de eletrólise, assim como o efeito da interação entre potencial aplicado e tempo de eletrólise.

A superfície de resposta mostrou um rendimento ótimo previsto de $60,5 \%$ para o potencial aplicado de $25 \mathrm{~V}$, tempo de eletrólise de 72 h e quantidade de eletrólito de $90 \%$.

\section{AGRADECIMENTOS:}

FM agradece à CAPES a bolsa concedida.

\section{REFERÊNCIAS:}

1. ABNT. ASSOCIAÇÃO BRASILEIRA DE NORMAS TÉCNICAS. NBR 10004: Resíduos sólidos - Classificação. Rio de Janeiro, 2004.

2. Ahmad T., Ahmad, K., Alam, M. J. Cleaner Prod, 2016, 124, 1.

3. ASTM D1193-06(2011), Standard Specification for Reagent Water, ASTM International, West Conshohocken, PA, 2011.

4. Baird, C. Química Ambiental, $2^{\mathrm{a}}$ ed. Porto Alegre: Bookman, 2002

5. Bard, A.J., Inzelt, G., Scholz, F. (Eds.) Electrochemical Dictionary, $1^{\text {a }}$ ed. Berlin, Heidelberg: Springer, 2008.

6. BRASIL. Lei $n^{\circ} 12305$, de 2 de agosto de 2010. Institui a Política Nacional de Resíduos Sólidos. Diário Oficial da República Federativa do Brasil, Brasília, 2ago 2010.

7. Cherifi, M., Hazaourli, S., Ziati, M. Phys. Procedia, 2009, 2, 1021.

8. Cherifi, M., Hazourli, S., Pontvianne, S., Leclerc, J.P., Lapicque, F. Desalination, 2011, 281, 263.

9. Cherifi, M., Boutemine, N., Laefer, D.F., Hazourli, S. C. R. Chim, 2016, 19, 511.

10. Ebbers, B., Ottosen, L.M., Jensen, P.E. Electrochim. Acta, 2015, 181, 90.

11. EPA - United States Environmental Protection Agency, 1996. "Method 3050B: Acid Digestion of Sediments, Sludges, and Soils," Revision 2.

12. Fadanelli, L.E.A., Wiecheteck, G.K. Rev. Eng. Tecnol, 2010, 2, 31. 
13. Ferreira, R.P., Moreira, A., Rassini, J.B. Toxidez de alumínio em culturas anuais, $1^{\mathrm{a}}$ ed. São Carlos: Embrapa, 2006.

14. Ferri, V., Ferro, S., Martínez-Huitle, C.A., Battisti, A. Electrochim. Acta, 2009, 54, 2108.

15. Julio, M., Santos, M.N.O., Oroski, F.I. Rev. Eng. Tecnol, 2009, 1, 14.

16. Kim, G.-N., Kim, S.-S., Park, U.-R., Moon, J.-K. Electrochim. Acta, 2015, 181, 233.

17. Lageman, R., Clarke, R.L., Pool, W. Eng. Geol, 2005, 77, 191.

18. Moreira, R.C.A., Guimarães, E.M., Boaventura, G.R., Momesso, A. M., Lima, G.L. Quim. Nova, 2009, 32, 2085.

19. Page, M.M., Page, C.L. J. Environ. Eng, 2002, 128, 208.

20. Portella, K.F., Andreoli, C. V., Hoppen, C., Sales, A., Baron, O. $22^{\circ}$ Congresso Brasileiro de Engenharia Sanitária e Ambiental, Joinvile, 2003.

21. Rosestolato, D., Bagatin, R., Ferro, S. Chem. Eng. J, 2015, 264, 16.

22. Sah, J.G., Chen, J. Y. J. Hazard. Mater, 1998, 58, 301.

23. Tak, B.-Y., Tak, B.-S., Kim, Y.-J., Park, Y.-J., Yoon, Y.-H., Min, G.-H. J. Ind. Eng. Chem, 2015, 28, 307.

24. Tartari, R., Diaz-Mora, N., Módenes, A.N., Pianaro, S. A. Ceramica, 2011, 57, 288.

25. Vaz, L. G. L., Klen, M.R.F., Veit, M.T., Silva, E. A., Barbiero, T. A., Bergamasco, R. Ecletica Quim, 2010, 35, 45.

26. Wang, J. -Y., Zhang, D. -S., Stabnikova, O., Tay, J. -H. J. Hazard. Mater, 2005, B124, 139.

27. Wang, J. -Y., Huang, X. -J., Kao, J. C. M., Stabniskova, O. J. Hazard. Mater, 2007, 144, 292.

28. Yu, J. -W., Neretnieks, I. J. Contam. Hydrol, 1997, 26, 291.

29. Yuan, C., Weng, C. -H. Chemosphere, 2006, 65, 88. 


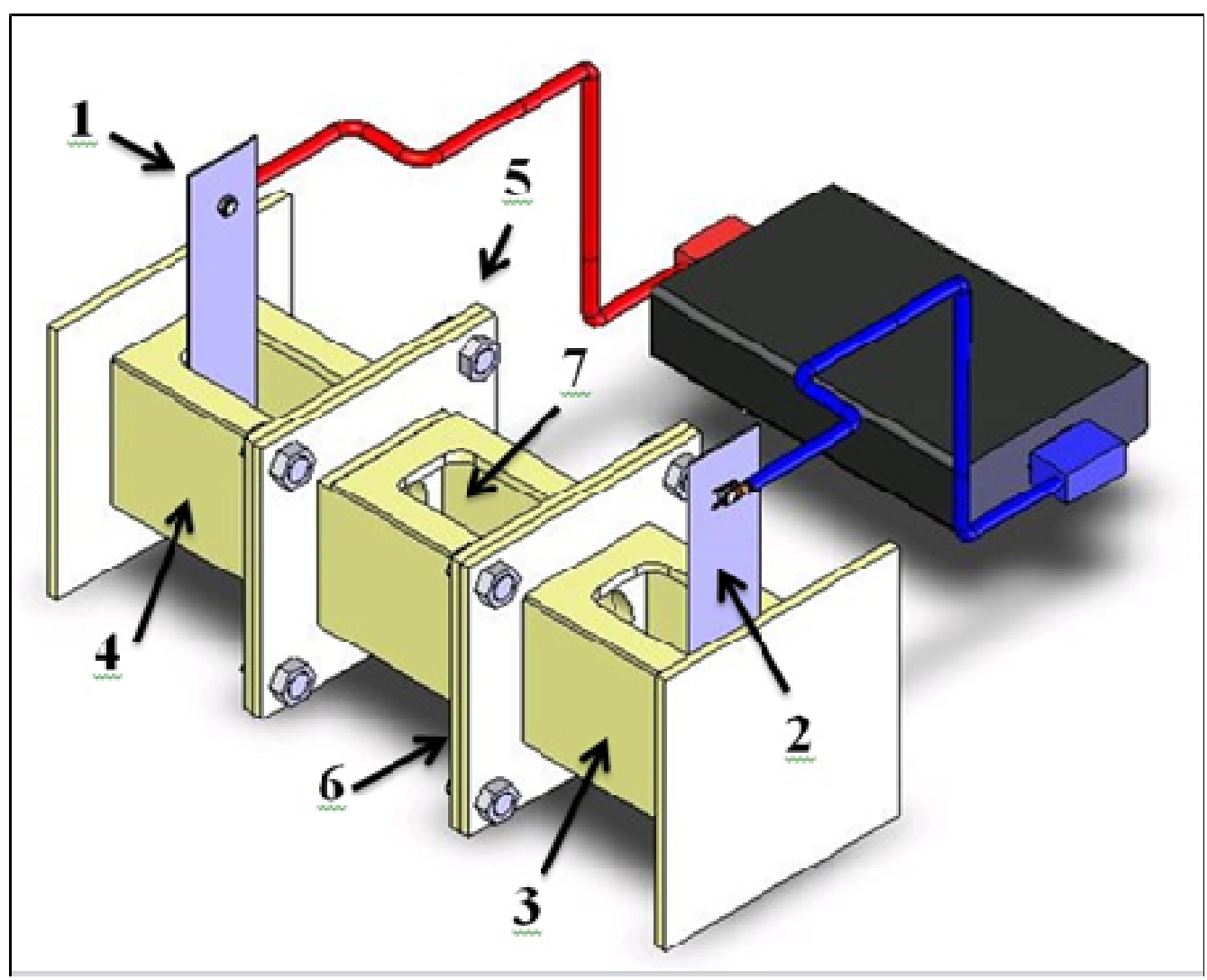

Figura 1. Desenho esquemático do arranjo experimental: 1: cátodo; 2: ânodo; 3: compartimento anódico; 4: compartimento catódico; 05: membrana catiônica; 06: membrana aniônica; 07: compartimento para o lodo contaminado 
A

Response surface for Remoção $\mathrm{Al}(1)$

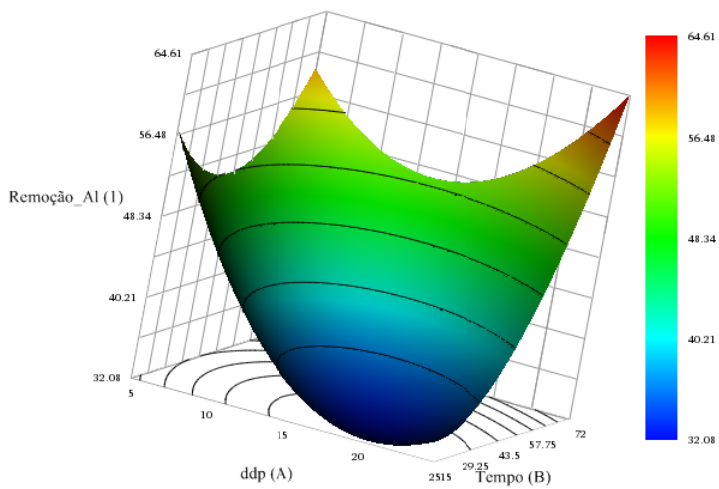

B

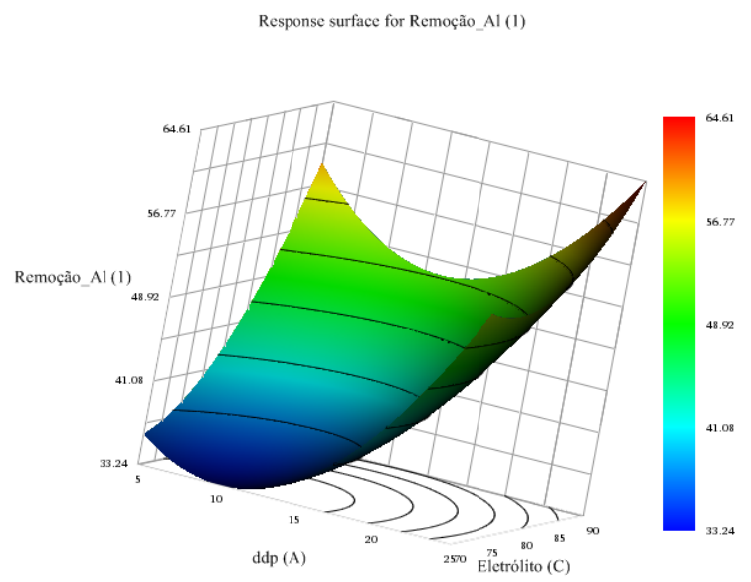

C

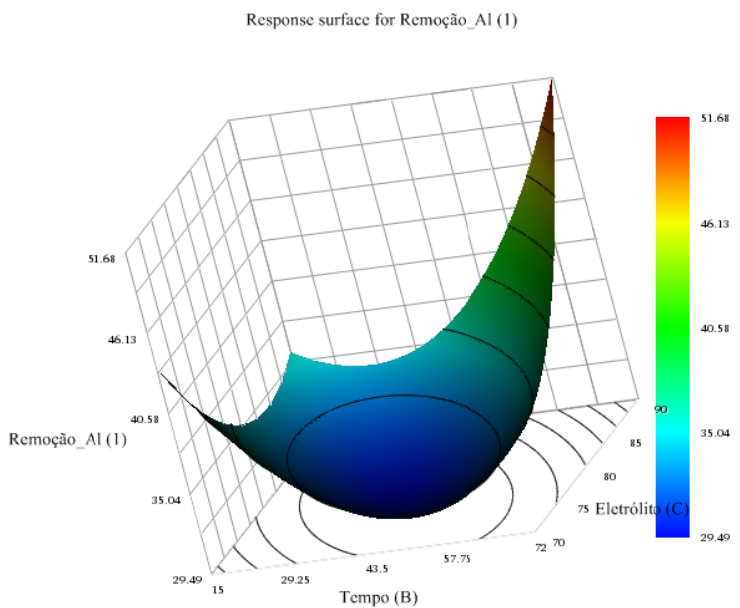

Figura 2. Superfícies de respostas para a máxima remoção de alumínio

PERIÓDICO TCHÊ QUÍMICA • www.periodico.tchequimica.com • Vol. 14 N. 28.

• ISSN 1806-0374 (impresso) • ISSN 1806-9827 (CD-ROM) • ISSN 2179-0302 (meio eletrônico) (C) 2017. Porto Alegre, RS. Brasil

The Periódico Tchê Quimica (ISSN: 1806-0374; 2179-0302) is an open-access journal since 2004. Journal DOI: 10.52571/PTQ. http://www.tchequimica.com.

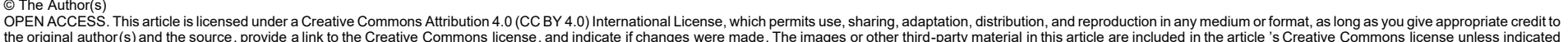
the original author(s) and the source, provide a link to the Creative Commons license, and indicate if changes were made. The images or other third-party material in this article are included in the article 's Creative Commons license unless indicated

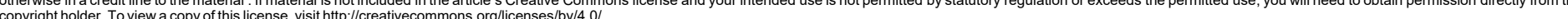

